\title{
Reproducibility and validity of dietary patterns identified using factor analysis among Chinese populations
}

\author{
Xin Hong ${ }^{1}$, Qing Ye ${ }^{1}$, Zhiyong Wang ${ }^{1}$, Huafeng Yang $^{1}$, Xupeng Chen ${ }^{1,2}$, Hairong Zhou ${ }^{1,2}$, \\ Chenchen Wang ${ }^{1,2}$, Wenjie Chu ${ }^{2}$, Yichao Lai ${ }^{3}$, Liuyuan Sun ${ }^{4}$, Youfa Wang ${ }^{5}$ and Fei $\mathrm{Xu}^{1,2 *}$ \\ ${ }^{1}$ Department of Non-communicable Disease Prevention, Nanjing Municipal Center for Disease Control E Prevention, \\ 2, Zizhulin, Nanjing 210003, People's Republic of China \\ ${ }^{2}$ Department of Epidemiology and Biostatistics, School of Public Health, Nanjing Medical University, Nanjing 211166, People's \\ Republic of China \\ ${ }^{3}$ Department of Non-communicable Disease Prevention, Qinhuai District Center for Disease Control E Prevention, Nanjing \\ 210029, People's Republic of China \\ ${ }^{4}$ Department of Non-communicable Disease Prevention, Liube District Center for Disease Control E Prevention, Nanjing \\ 211500, People's Republic of China \\ ${ }^{5}$ Department of International Health, Bloomberg School of Public Health, Johns Hopkins Global Center for Childhood Obesity, \\ Johns Hopkins University, Baltimore, MD 21205, USA
}

(Submitted 2 March 2016 - Final revision received 18 May 2016 - Accepted 27 May 2016 - First published online 13 July 2016)

\section{Abstract}

In the present study, we evaluated the reproducibility and validity of dietary patterns among Chinese adult populations. A random subsample of 203 participants (aged 31-80 years) from a community-based nutrition and health survey was enrolled. An eighty-seven-item FFQ was administered twice (FFQ1 and FFQ2) 1 year apart; four 3 consecutive day, 24-h dietary recalls (24-HDR, as a reference method) were performed between the administrations of the two FFQ every 3 months. Dietary patterns from three separate dietary sources were derived using factor analysis based on twenty-eight predefined food groups. Comparisons between dietary pattern scores were made by using Pearson's or intraclass correlation coefficients (ICC), cross-classification analysis, weighted $\kappa$ statistic and Bland-Altman plots; the four major dietary patterns identified from FFQ1, FFQ2 and 24-HDR were similar. Regarding reproducibility, ICC for $z$-scores between FFQ1 and FFQ2 were all $>0.6$ for dietary patterns. The 'animal and plant protein' pattern had the highest ICC of $0 \cdot 870$. For validity, the adjusted Pearson's correlation coefficients for dietary pattern $z$-scores between two FFQ and the mean of four 3 consecutive day 24-HDR ranged from 0.387 for the 'Chinese traditional' pattern to 0.838 for the 'animal and plant protein' pattern. More than $75 \%$ of the participants were classified into the same or adjacent quartile, and $<5 \%$ were misclassified into opposite quartiles. The weighted $\kappa$ ranged from 0.259 to 0.680 . Bland-Altman plots indicated that no significant deviation was found between two dietary assessment methods. Our findings indicate a good reasonable reproducibility and a reasonable validity of dietary patterns derived by factor analysis in China.

Key words: Reliability: Validity: Dietary patterns: Factor analyses: China

Epidemiological studies have suggested that dietary pattern analysis is a useful method for studying the role of diet in relation to health outcomes or disease risk. Dietary pattern analysis has been used increasingly as an alternative method to traditional analysis because it takes into account the diet's overall effects, reflecting more closely the real-world habits ${ }^{(1,2)}$.

The following three main approaches have been used to define dietary patterns: factor analysis, cluster analysis and dietary indices. Factor analysis is a multivariate statistical reduction technique that aggregates specific food groups based on analyses of the correlation-covariance matrix of a number of food items ${ }^{(3)}$. The continuous nature of factor analysis has been seen to be advantageous over other methods ${ }^{(4)}$. Factor analysis was therefore commonly used to derive dietary patterns.

However, several subjective or arbitrary decisions can be made during factor analysis, including the consolidation of food items into food groups, the number of factors to extract, the method of rotation and the labelling of the components ${ }^{(5)}$. Furthermore, dietary patterns can be population specific, such that it is essential to identify dietary patterns in a specific study population of interest ${ }^{(1,6)}$, such as the Chinese population. In the past two decades, China has experienced a significant

Abbreviations: 24-HDR, 24-h dietary recalls; FA, factor analysis; ICC, intraclass correlation coefficients; LOA, limits of agreement; m24-HDR, mean of four 3 consecutive day 24-HDR; mFFQ, mean of two FFQ.

*Corresponding author: F. Xu, email frankxufei@163.com 
nutrition transition from the traditional Chinese diet to a Western diet pattern, with an increase in consumption of red meats, eggs and oils and a decrease in fruit and vegetable intakes.

Most dietary pattern studies have used FFQ to estimate dietary intakes, as they are easy to administer, comparatively inexpensive, and they can assess long-term dietary habits in large populations. However, FFQ are sensitive to the diverse lifestyle, eating habits and dietary preferences in the population concerned ${ }^{(7)}$. Dietary recalls may be superior to FFQ and have been frequently used as a reference method in many Chinese validation studies ${ }^{(8-10)}$. To date, some foreign studies ${ }^{(11-18)}$ have been conducted to examine the validity of dietary patterns derived from FFQ using factor analysis. Unfortunately, no similar studies have been reported in China, with different culture-specific dietary habits.

The purpose of the present study was to examine the reproducibility and validity of dietary patterns derived from factor analysis among Chinese populations. The reproducibility was assessed by comparing the dietary pattern scores between two FFQ administered 1 year apart, and the validity was assessed by comparing the dietary pattern scores between FFQ and 24-h dietary recalls (24-HDR) as a reference method at 3 -month intervals during the period of 1 year.

\section{Methods}

\section{Study population}

The present study was conducted using a subsample of the community-based, cross-sectional, nutrition and health survey in Nanjing, the capital of Jiangsu Province of China. The detailed study recruitment methods have been described previously $^{(19)}$. In brief, a multi-stage random sampling method was adopted. First, we randomly selected two districts (one urban and one suburban). Next, three streets/towns from each chosen district were randomly selected. Finally, one community from each chosen street/town was randomly selected. This resulted in a total number of six communities. Of 2030 participants of the nutrition and health survey, a random sample of 250 members was invited to participate in the present study. Sample size of the present study was calculated according to subjects per food group ratios of $7: 1^{(18)}$. Inclusion criteria were as follows: local resident for at least 5 years, aged 30 years or above, free of chronic non-communicable diseases requiring a special diet and not on a weight-reduction diet. Among the 250 selected residents, 248 were eligible to participate and 223 agreed to take part in the survey (response rate: $89.9 \%$ ).

The Ethics Board of Nanjing Municipal Center for Disease Control and Prevention reviewed and approved the study protocol, and written informed consent was obtained from each participant before inclusion.

\section{Study design}

The study design with time frame is shown in Fig. 1. The study stared in June 2014 and ended in May 2015. Each participant completed the same FFQ twice - the first FFQ (FFQ1) was administrated at baseline and the second FFQ (FFQ2) was administrated 1 year later; four 3 consecutive day 24-HDR were collected between the administrations of the two FFQ every 3 months during a period of 1 year (a total of twelve 24-HDR). The first 3 consecutive day 24-HDR was performed 1 month after FFQ1, and the last 3 consecutive day 24-HDR was performed 1 month before FFQ2. We excluded participants who failed to provide two completed FFQ ( $n$ 6), did not complete four 3-d 24-HDR ( $n$ 9) or had extreme values for total energy intake $(<2092 \mathrm{~kJ} / \mathrm{d} \quad(<500 \mathrm{kcal} / \mathrm{d})$ or $>20920 \mathrm{~kJ} / \mathrm{d}$ ( $>5000 \mathrm{kcal} / \mathrm{d}), n$ 5). Finally, 203 subjects (81.9\%) were included in the data analysis.

\section{Dietary assessment}

A semi-quantitative FFQ was used to estimate habitual dietary intakes over the previous year. The reproducibility and validity of the FFQ used in this study have been published elsewhere ${ }^{(19)}$. The FFQ included eighty-seven food items and

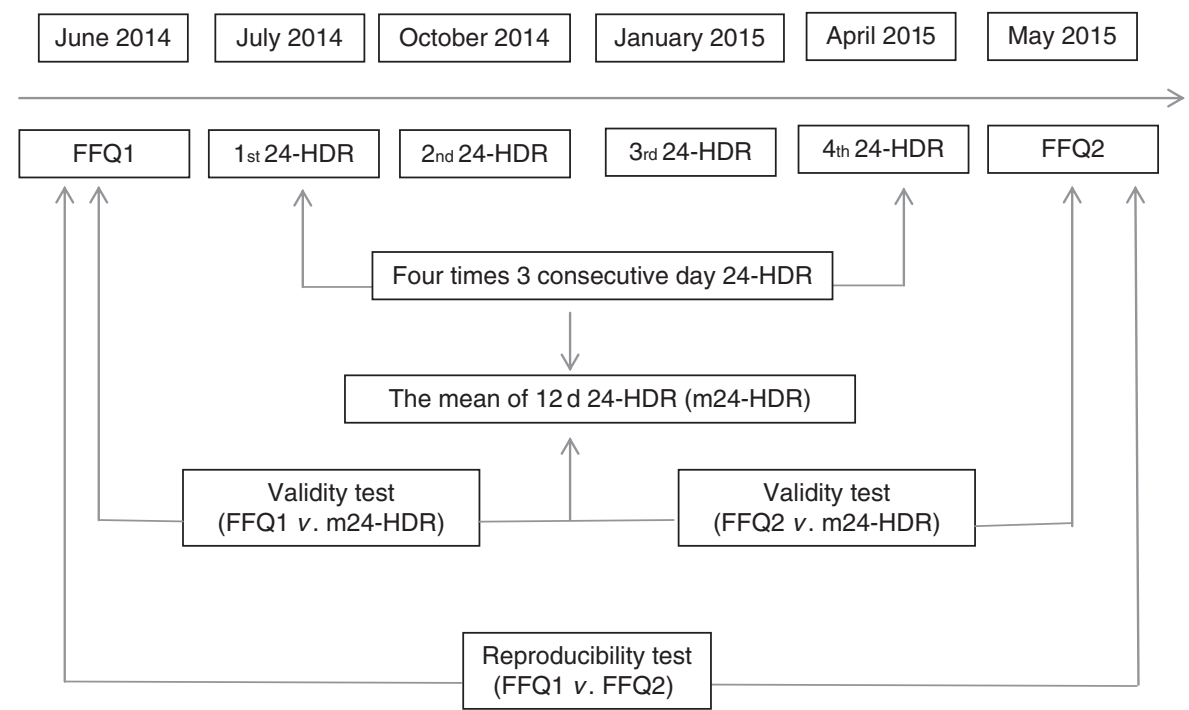

Fig. 1. Study design and time frame used in the present study. 24-HDR, 24-h dietary recalls; m24-HDR, mean of four 3 consecutive day 24-HDR. 
twelve food categories (grains and products; red meat (pork, beef, mutton); poultry meat; fish and shrimp; eggs; dairy products; soya-based foods; vegetables; fruits; beverages; alcohol; snacks/desserts), which covered $90 \%$ of the commonly consumed foods in Nanjing. For each food item, participants were asked to recall the frequency of consumption (daily, weekly, monthly, annually or never) and the amount of consumption each time in a common unit of weight in China (1 liang $=50 \mathrm{~g}$ ) or in millilitre over the past 12 months. For seasonal vegetables and fruits, participants were asked to recall how often they ate these foods during the season. Individual consumption of food items was converted to grams per day for further analysis.

Owing to the small number of subjects ( $n$ 203) relative to the number of food items, and to reduce the complexity of the data, we collapsed the initial eighty-seven food items into twentyeight predefined food groups (Appendix 1). The grouping scheme was based on the similarity of nutrient profiles or culinary usage among the foods ${ }^{(13,20)}$.

In total, four 3 consecutive day (including 2 weekdays and 1 weekend day in a usual week) 24-HDR were collected at intervals of 3 months during the 1-year period. Each participant was asked to provide the name and amount of all foods consumed during the previous $24 \mathrm{~h}$. If the previous day was a special day, such as feast or travel days, food consumption of the day before the $24 \mathrm{~h}$ was recorded or another day was chosen to interview the participant by telephone. The amounts of different food items that were mixed in one dish were recorded, respectively. The recalled food items were assigned to the corresponding food groups as defined by the FFQ. The Chinese Food Composition Tables ${ }^{(21)}$ were used to estimate the intake of energy $(\mathrm{kJ} / \mathrm{d}(\mathrm{kcal} / \mathrm{d}))$ and key nutrients from each food group consumed by 24-HDR. All values obtained for key nutrient intake were adjusted for total energy intake using the regression residual method ${ }^{(22)}$. The mean of four 3 consecutive day 24-HDR (m24-HDR) data was used as the standard to measure the validity of the FFQ.

Trained interviewers from the local Center for Disease Control and Prevention administered the two FFQ and four 3-d 24-HDR by face-to-face interviews. All diet information were collected and checked after completion. Any implausible or ambiguous information would be further verified and obtained from the participants. Each participant had the same interviewer during the study period.

\section{Dietary pattern analysis}

Exploratory factor analysis (FA) was used to identify major dietary patterns based on a set of twenty-eight predefined food groups; FA was performed separately for FFQ1, FFQ2 and m24-HDR food groups. Factors were rotated with varimax rotation to maintain uncorrelated factors and enhance interpretability. A combined evaluation of the eigenvalues, scree plot test and factor interpretability was used in determining the number of retained factors ${ }^{(11,23)}$. Factor loadings were interpreted as correlation coefficients between food groups and dietary patterns. Food groups with positive loadings contributed to the dietary pattern, and food groups with negative loadings were inversely associated with the dietary pattern. Food groups with absolute factor loadings $\geq 0.30$ were considered as significantly contributing to the pattern ${ }^{(24)}$. The patterns were labelled according to food groups with high loadings in each dietary pattern. The sum of the squares of the respective factor loadings over all retained factors represented the percentage of variance that was explained by the final factors. Factor scores for each pattern were calculated as the sum of the products of the factor loading coefficients and the standardised daily intake of each food group ${ }^{(24)}$.

\section{Statistical analyses}

The Kaiser-Meyer-Olkin (KMO) measure of sampling adequacy $(>0.6)$ and Bartlett's test of sphericity $(P<0.05)$ were used to determine the data suitability for FA. Three methods were used to examine the reliability and validity of dietary patterns.

First, the reproducibility and validity were assessed by comparing dietary pattern scores between FFQ1 and FFQ2, and between two FFQ and m24-HDR, respectively, using Pearson's or intraclass correlation coefficients (ICC), cross-classification analysis and weighted $\kappa(K w)$ statistic. Cross-classification (quartiles method) analysis was conducted to classify the participants into same, adjacent, one quartile apart or opposite quartiles. The inter-rater agreement of the two assessment methods was analysed by $K w$. ICC $>0.4$ indicated good agreement ${ }^{(25)}$. Pearson's correlation coefficients of $0.5-0.7$ were considered $\operatorname{good}^{(26)}$. Values of $K w>0.4$ indicated moderate agreement $^{(26)}$.

Second, a Bland-Altman plot was constructed to assess the agreement of dietary pattern scores between different dietary sources. The plots showed the difference between each individual's $z$-scores derived from the mean of two FFQ (mFFQ) and $\mathrm{m} 24-\mathrm{HDR}$ (mFFQ-m24-HDR) against their averages $((\mathrm{mFFQ}+\mathrm{m} 24-\mathrm{HDR}) / 2)^{(27)}$. The mean differences and the $95 \%$ limits of agreement (LOA, calculated as mean differences $\pm 1.96 \mathrm{SD}$ ) were used to summarise agreement at the population level.

Third, Pearson's correlation coefficients were used to compare energy-adjusted nutrient intakes estimated by m24-HDR with dietary pattern scores derived from FFQ and m24-HDR.

All statistical analyses were performed using SPSS software version 20.0 (IBM) and MedCalc version 11.4. All tests were two-tailed, and a $P$ value $<0.05$ was considered statistically significant.

\section{Results}

\section{Study sample characteristics}

Among 203 participants, about 48.8\% were males and 92.2\% were married. Their mean age was 50.4 (SD 12.2) years (range $31-80$ years); the mean BMI was $23 \cdot 1$ (sD $2 \cdot 8$ ) kg/m² ; and $79.5 \%$ had educational qualification of junior high school or above. The proportion of current smokers and drinkers was 22.0 and $28.8 \%$, respectively. There were no differences in baseline characteristics between the subsample ( $n$ 203) and the entire population ( $n$ 2030) (Appendix 2). 
Table 1. Factor-loading matrix for four major dietary patterns* identified from FFQ1, FFQ2 and the mean of four 3 consecutive day 24-HDR (m24-HDR) in the subsamples ( $n$ 203)

\begin{tabular}{|c|c|c|c|c|c|c|c|c|c|c|c|c|}
\hline \multirow[b]{2}{*}{ Food groups } & \multicolumn{4}{|c|}{ FFQ1 } & \multicolumn{4}{|c|}{ FFQ2 } & \multicolumn{4}{|c|}{ m24-HDR } \\
\hline & $\begin{array}{l}\text { Animal and } \\
\text { plant }\end{array}$ & $\begin{array}{l}\text { Nuts and } \\
\text { sweets }\end{array}$ & $\begin{array}{l}\text { Chinese } \\
\text { traditional }\end{array}$ & $\begin{array}{l}\text { Beverage and } \\
\text { alcohol }\end{array}$ & $\begin{array}{c}\text { Animal and } \\
\text { plant }\end{array}$ & $\begin{array}{l}\text { Nuts and } \\
\text { sweets }\end{array}$ & $\begin{array}{l}\text { Chinese } \\
\text { traditional }\end{array}$ & $\begin{array}{l}\text { Beverage and } \\
\text { alcohol }\end{array}$ & $\begin{array}{l}\text { Animal and } \\
\text { plant }\end{array}$ & $\begin{array}{l}\text { Nuts and } \\
\text { sweets }\end{array}$ & $\begin{array}{l}\text { Chinese } \\
\text { traditional }\end{array}$ & $\begin{array}{l}\text { Beverage and } \\
\text { alcohol }\end{array}$ \\
\hline Poultry meats & $0.767 \dagger$ & 0.045 & -0.246 & $0.313 \dagger$ & $0.841 \dagger$ & 0.032 & -0.050 & 0.217 & $0.630 \dagger$ & 0.012 & -.021 & $0.360 \dagger$ \\
\hline Fish and shrimp & $0.748 \dagger$ & -0.014 & -0.048 & 0.115 & $0.807 \dagger$ & 0.014 & -0.020 & 0.058 & $0.656 \dagger$ & -0.002 & 0.046 & 0.098 \\
\hline Bean curd & $0.727 \dagger$ & -0.034 & 0.045 & 0.016 & $0.762 \dagger$ & 0.051 & 0.003 & 0.096 & $0.347 \dagger$ & -0.241 & 0.245 & 0.132 \\
\hline Dry vegetables & $0.720 \dagger$ & 0.206 & 0.093 & -0.088 & $0.761 \dagger$ & 0.032 & 0.099 & -0.115 & $0.732 \dagger$ & 0.207 & 0.070 & -0.249 \\
\hline Livestock meats & $0.706 \dagger$ & 0.075 & -0.259 & 0.273 & $0.717 \dagger$ & 0.132 & 0.072 & 0.049 & $0.600 \dagger$ & $-0.317 \dagger$ & 0.162 & 0.108 \\
\hline Dry bean & $0.666 \dagger$ & -0.017 & 0.273 & $-0.316 \dagger$ & $0.804 \dagger$ & 0.088 & 0.108 & 0.006 & $0.728 \dagger$ & 0.112 & -.013 & -0.145 \\
\hline Other soyabean products & $0.642 \dagger$ & 0.166 & 0.197 & -0.171 & $0.655 \dagger$ & 0.106 & 0.084 & -0.046 & $0.437 \dagger$ & 0.074 & 0.251 & -0.223 \\
\hline Eggs & $0.594 \dagger$ & 0.259 & 0.070 & 0.268 & $0.659 \dagger$ & 0.246 & 0.207 & 0.215 & $0.653 \dagger$ & 0.020 & -0.019 & 0.116 \\
\hline Fresh fruits & $0.381 \dagger$ & $0.362 \dagger$ & 0.109 & 0.066 & $0.590 \dagger$ & 0.141 & $0.334 \dagger$ & 0.031 & $0.519 \dagger$ & 0.215 & 0.251 & -0.006 \\
\hline Low-fat dairy products & $0.318 \dagger$ & $0.304 \dagger$ & 0.164 & 0.005 & $0.415 \dagger$ & $0.448 \dagger$ & 0.082 & $0 \cdot 168$ & $0.313 \dagger$ & 0.122 & -0.310 & 0.091 \\
\hline Tea or coffee & $0.310 \dagger$ & -0.019 & -0.160 & -0.045 & 0.282 & -0.071 & -0.156 & -0.090 & 0.264 & -0.060 & -0.092 & 0.294 \\
\hline Nuts & 0.033 & $0.750 \dagger$ & 0.059 & -0.065 & 0.010 & $0.673 \dagger$ & 0.168 & 0.025 & 0.181 & $0.369 \dagger$ & 0.001 & 0.108 \\
\hline Sweets and desserts & -0.021 & $0.743 \dagger$ & 0.044 & 0.023 & 0.056 & $0.749 \dagger$ & 0.006 & 0.271 & 0.140 & $0.564 \dagger$ & 0.221 & 0.260 \\
\hline Snacks & 0.098 & $0.666 \dagger$ & -0.020 & -0.028 & 0.053 & $0.489 \dagger$ & 0.188 & -0.173 & 0.051 & $0.361 \dagger$ & 0.097 & 0.058 \\
\hline Other grains and products & 0.113 & $0.377 \dagger$ & $0.580 \dagger$ & -0.067 & 0.039 & 0.146 & $0.670 \dagger$ & -0.039 & 0.079 & $0.334 \dagger$ & $0.353 \dagger$ & -0.137 \\
\hline Potatoes & 0.129 & $0.301 \dagger$ & $0.579 \dagger$ & 0.125 & 0.072 & 0.060 & $0.695 \dagger$ & 0.075 & -0.024 & 0.096 & $0.386 \dagger$ & -0.060 \\
\hline Fresh vegetables & 0.163 & 0.143 & $0.552 \dagger$ & -0.028 & 0.250 & 0.066 & $0.430 \dagger$ & -0.216 & -0.036 & -0.067 & 0.493 & -0.217 \\
\hline Fried food & 0.032 & -0.143 & $0.519 \dagger$ & 0.223 & -0.060 & -0.038 & $0.574 t$ & $0.300 \dagger$ & 0.004 & 0.078 & $0.437 \dagger$ & 0.201 \\
\hline High-fat dairy products & $0.359 \dagger$ & 0.002 & $0.482 \dagger$ & 0.199 & 0.286 & -0.071 & $0.466 \dagger$ & $0.374 \dagger$ & 0.069 & $0.307 \dagger$ & $0.486 \dagger$ & 0.068 \\
\hline Wheat and products & 0.019 & $0.383 \dagger$ & $0.440 \dagger$ & -0.027 & 0.060 & 0.074 & $0.620 \dagger$ & -0.070 & 0.165 & -.048 & $0.419 \dagger$ & 0.053 \\
\hline Rice and products & 0.236 & -0.292 & $0.418 \dagger$ & 0.132 & $0.321 \dagger$ & $-0.306 \dagger$ & $0.454 \dagger$ & -0.035 & 0.071 & -0.262 & $0.511 \dagger$ & 0.257 \\
\hline Pickled vegetables & -0.158 & -0.236 & $0.330 \dagger$ & -0.228 & -0.156 & - & $0.513 \dagger$ & -0.081 & -0.085 & -0.248 & $0.544 \dagger$ & -0.037 \\
\hline Sodas & 0.024 & -0.003 & 0.120 & $0.592 \dagger$ & -0.031 & 0.102 & -0.027 & $0.702 \dagger$ & -0.086 & 0.073 & 0.055 & 0.460 \\
\hline Juice & 0.103 & 0.034 & -0.020 & $0.558 \dagger$ & 0.058 & 0.103 & -0.091 & $0.718 \dagger$ & -0.081 & 0.272 & 0.171 & 0.507 \\
\hline Beer & 0.011 & -0.162 & -0.073 & $0.465 \dagger$ & 0.042 & -0.258 & -0.077 & $0.532 \dagger$ & 0.067 & 0.137 & 0.125 & 0.591 \\
\hline Wine & 0.089 & -0.033 & $0.312 \dagger$ & $0.392 \dagger$ & 0.086 & -0.189 & 0.269 & $0.418 \dagger$ & 0.179 & -.072 & -0.106 & 0.344 \\
\hline Processed meats & 0.017 & 0.020 & 0.063 & $0.369 \dagger$ & 0.011 & 0.169 & 0.051 & $0.560 \dagger$ & -0.069 & -0.126 & 0.059 & 0.363 \\
\hline Liquor & -0.068 & -0.180 & 0.079 & $0.306 \dagger$ & -0.075 & $-0.365 \dagger$ & 0.077 & $0.313 \dagger$ & 0.020 & $-0.516 \dagger$ & -0.120 & 0.375 \\
\hline Eigenvalue & 4.990 & 2.571 & 2.489 & 1.943 & 5.946 & 2.006 & 2.640 & 2.890 & 3.905 & $2 \cdot 160$ & 1.889 & 1.759 \\
\hline Variance explained (\%) & $16 \cdot 6$ & 8.6 & $8 \cdot 3$ & 6.5 & $19 \cdot 8$ & $6 \cdot 7$ & 8.8 & 9.6 & $13 \cdot 0$ & $7 \cdot 2$ & $6 \cdot 3$ & 5.9 \\
\hline Total variance (\%) & \multicolumn{4}{|c|}{$40 \cdot 0$} & \multicolumn{4}{|c|}{44.9} & \multicolumn{4}{|c|}{32.4} \\
\hline
\end{tabular}

* The test for suitability of factor analysis: the Kaiser-Meyer-Olkin measure of sampling adequacy was 0.734 for FFQ1, 0.806 for FFQ2 and 0.640 for m24-HDR, and $P$ values for Bartlett's test of sphericity were all $<0.001$.

$\dagger$ The factor loadings were $>0.30$. 
Dietary patters identified in the two FFQ and mean of four 3 consecutive day $24-H D R$

The KMO measure of sampling adequacy was 0.734 for FFQ1, 0.806 for FFQ2 and 0.640 for $\mathrm{m} 24-\mathrm{HDR}$, and $P$ values for Bartlett's test of sphericity were all $<0 \cdot 001$. Using FA, four major dietary patterns were extracted from FFQ1, FFQ2 and m24-HDR (Table 1). These four derived patterns were relatively similar from three dietary sources. Factor 1 , which loaded heavily on poultry meats, fish and shrimp, bean curd, livestock meats, dry bean and other soyabean products, was labelled the 'animal and plant protein' pattern. Factor 2, with high loadings for nuts, sweets and desserts and snacks, was labelled the "nuts and sweets' pattern. Factor 3, which was rich in other grains and products, potatoes, fresh vegetables, fried food, high-fat dairy products, wheat and products, rice and products, and pickled vegetables, was labelled the 'Chinese traditional' pattern. Factor 4, characterised by higher intake of sodas, juice, beer, wine, processed meats and liquor, was labelled the beverage and alcohol' pattern. Overall, the total percentage of variance explained by the four patterns derived from FFQ1, FFQ2 and $\mathrm{m} 24-\mathrm{HDR}$ was $40 \cdot 0,44.9,32.4 \%$, respectively. In addition, four similar dietary patterns were also identified in the overall samples (Appendix 3).

\section{Correlations and agreement between dietary pattern} z-scores

Regarding reproducibility, ICC for dietary pattern $z$-scores between FFQ1 and FFQ2 were $>0.6$ for all four patterns. The 'animal and plant protein' pattern had the highest ICC of 0.870 (Table 2). For validity, the adjusted Pearson's correlation coefficients for dietary pattern $z$-scores between two FFQ and m24HDR ranged from 0.387 for the 'Chinese traditional' pattern to 0.838 for the 'animal and plant protein' pattern.

When the four dietary pattern scores were categorised into quartiles, the ranges of agreement rates for the same or adjacent quartile classifications were $75 \cdot 6-95.5 \%$, when derived from the two FFQ and the m24-HDR. Extreme misclassification into opposite quartiles was $<5.0 \%$ (Table 3 ). The $K w$ ranged from $0 \cdot 259$ to $0 \cdot 680$

The Bland-Altman plots of all dietary patterns are presented in Fig. 2-5. The mean agreement between the dietary pattern $z$-scores derived from the mFFQ and the m24-HDR were not significantly different from zero in all comparisons. The mean differences were 0.0 (95\% LOA $-1.03,1.04)$ for the 'animal and plant protein' pattern, -0.0 (95\% LOA $-1.7,1.6)$ for the 'nuts and sweets' pattern, $-0 \cdot 1$ (95\% LOA $-2 \cdot 0,1 \cdot 8)$ for the 'Chinese traditional' pattern and $-0.2(95 \% \mathrm{LOA}-1 \cdot 9,1.5)$ for the 'beverage and alcohol' pattern between mFFQ and m24-HDR.

\section{Correlations between dietary pattern z-scores and nutrient intakes}

Correlations between energy-adjusted nutrient intakes from the dietary recalls and dietary pattern scores derived from FFQ1, FFQ2 and $\mathrm{m} 24-\mathrm{HDR}$ are shown in Table 4. The majority of statistically significant correlations were consistent for the FFQ and m24-HDR. In particular, the 'animal and plant protein' pattern was positively correlated with intakes of protein, carbohydrates, fibre, vitamin $\mathrm{A}$, retinol, thiamine, riboflavin, niacin, vitamin $\mathrm{E}, \mathrm{Ca}, \mathrm{P}, \mathrm{K}, \mathrm{Mg}, \mathrm{Fe}, \mathrm{Zn}$, Se and $\mathrm{Cu}$, and was negatively correlated with intakes of total fat and cholesterol. In contrast, the 'Chinese traditional' pattern was negatively correlated with intakes of vitamin A, carotene, niacin, vitamin C, $\mathrm{Ca}, \mathrm{P}, \mathrm{Na}, \mathrm{Zn}$ and $\mathrm{Mn}$. The 'beverage and alcohol' patterns were positively correlated with intakes of total fat and cholesterol and negatively correlated with intakes of retinol, thiamine and Se.

\section{Discussion}

To our knowledge, the present study is perhaps the first one to assess the reproducibility and validity of dietary patterns identified by FA derived from FFQ in comparison with dietary recalls in a Chinese population. In a random subsample of 203 subjects, four major dietary patterns were identified using FA - that is, the 'animal and plant protein' pattern, the 'nuts and sweets' pattern, the 'Chinese traditional' pattern and the 'beverage and alcohol' pattern. These four derived patterns were qualitatively similar across three sources of dietary data obtained from the two FFQ and the means of twelve 24-HDR. For all dietary patterns, factor loadings of the FFQ and m24-HDR food groups were partly different. This might be due to methodological differences between dietary assessment methods ${ }^{(28,29)}$, random statistical variation and different assessment periods as mentioned previously ${ }^{(12,13,15,17,18)}$. The patterns identified in the present study were similar to previous findings in China ${ }^{(30-32)}$.

The correlations of the dietary pattern $z$-scores between FFQ1 and FFQ2 revealed good reliability, and the correlations of the dietary pattern $z$-scores between the two FFQ and the $\mathrm{m} 24-\mathrm{HDR}$ represented a reasonable comparative validity of four major dietary patterns derived by FA using the data of FFQ in a Chinese population. In this study, the 24-h recall

Table 2. Correlation coefficients for dietary pattern $z$-scores derived from FFQ1, FFQ2 and the mean of four 3 consecutive day 24-HDR (m24-HDR) in the subsamples $(n 203)^{\star}$

\begin{tabular}{lcccr}
\hline & Animal and plant & Nuts and sweets & Chinese traditional & Beverage and alcohol \\
\hline FFQ1 v. FFQ2† & 0.870 & 0.649 & 0.731 & 0.669 \\
FFQ1 v. m24-HDR & 0.838 & 0.440 & 0.387 & 0.440 \\
FFQ2 v. m24-HDR $\ddagger$ & 0.748 & 0.451 & 0.486 & 0.479 \\
\hline
\end{tabular}

${ }^{*}$ All correlations were statistically significant $(P<0.001)$.

$\dagger$ Values were intraclass correlation coefficients.

¥ Values were Pearson’s correlation coefficients adjusted for energy intake using the residual method. 
Table 3. Percentage agreement and $k$ statistic for dietary pattern $z$-scores derived from FFQ1, FFQ2 and the mean of four 3 consecutive day 24-HDR (m24-HDR) in the subsamples ( $n$ 203)

\begin{tabular}{|c|c|c|c|c|c|c|c|c|c|c|c|c|c|c|c|}
\hline \multirow[b]{2}{*}{ Dietary patterns } & \multicolumn{5}{|c|}{ FFQ1 v. FFQ2 } & \multicolumn{5}{|c|}{ FFQ1 v. m24-HDR } & \multicolumn{5}{|c|}{ FFQ2 v. m24-HDR } \\
\hline & $\begin{array}{c}\text { Same } \\
\text { quartile }\end{array}$ & $\begin{array}{l}\text { Adjacent } \\
\text { quartile }\end{array}$ & $\begin{array}{l}\text { One quartile } \\
\text { apart }\end{array}$ & $\begin{array}{l}\text { Opposite } \\
\text { quartile }\end{array}$ & $K w$ & $\begin{array}{l}\text { Same } \\
\text { quartile }\end{array}$ & $\begin{array}{l}\text { Adjacent } \\
\text { quartile }\end{array}$ & $\begin{array}{l}\text { One quartile } \\
\text { apart }\end{array}$ & $\begin{array}{l}\text { Opposite } \\
\text { quartile }\end{array}$ & $K w$ & $\begin{array}{l}\text { Same } \\
\text { quartile }\end{array}$ & $\begin{array}{l}\text { Adjacent } \\
\text { quartile }\end{array}$ & $\begin{array}{l}\text { One quartile } \\
\text { apart }\end{array}$ & $\begin{array}{c}\text { Opposite } \\
\text { quartile }\end{array}$ & $K w$ \\
\hline $\begin{array}{l}\text { Animal and } \\
\text { plant }\end{array}$ & $66 \cdot 3$ & $29 \cdot 2$ & 3.5 & 1.0 & 0.680 & $47 \cdot 0$ & $42 \cdot 7$ & $9 \cdot 1$ & $1 \cdot 2$ & 0.481 & $45 \cdot 4$ & $43 \cdot 2$ & $9 \cdot 2$ & $2 \cdot 2$ & 0.466 \\
\hline $\begin{array}{l}\text { Nuts and } \\
\text { sweets }\end{array}$ & $42 \cdot 4$ & 37.5 & $15 \cdot 6$ & 4.5 & 0.340 & $35 \cdot 7$ & 40.5 & $19 \cdot 5$ & $4 \cdot 3$ & 0.283 & $36 \cdot 8$ & $42 \cdot 7$ & $18 \cdot 4$ & $2 \cdot 1$ & 0.379 \\
\hline $\begin{array}{l}\text { Chinese } \\
\text { traditional }\end{array}$ & $52 \cdot 2$ & $39 \cdot 0$ & $7 \cdot 8$ & 1.0 & 0.576 & $32 \cdot 4$ & $43 \cdot 8$ & $19 \cdot 5$ & $4 \cdot 3$ & 0.282 & $36 \cdot 8$ & $45 \cdot 4$ & $15 \cdot 6$ & $2 \cdot 2$ & 0.355 \\
\hline $\begin{array}{l}\text { Beverage and } \\
\text { alcohol }\end{array}$ & $50 \cdot 2$ & 37.6 & 7.8 & 4.4 & 0.527 & $35 \cdot 1$ & 40.5 & $19 \cdot 6$ & 4.8 & 0.259 & $36 \cdot 8$ & 38.9 & 19.4 & 4.9 & 0.295 \\
\hline
\end{tabular}

$K w$, weighted $\kappa$.
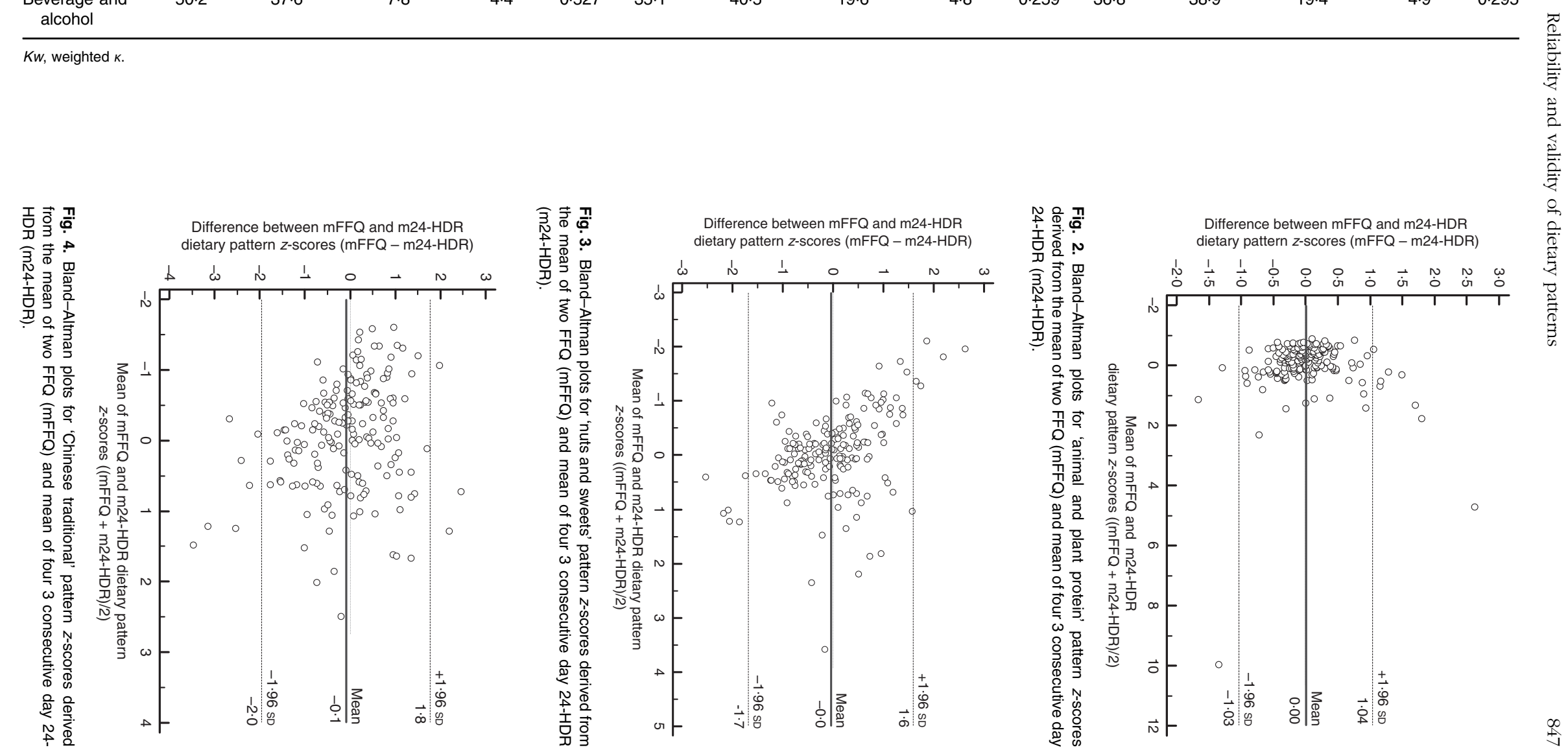


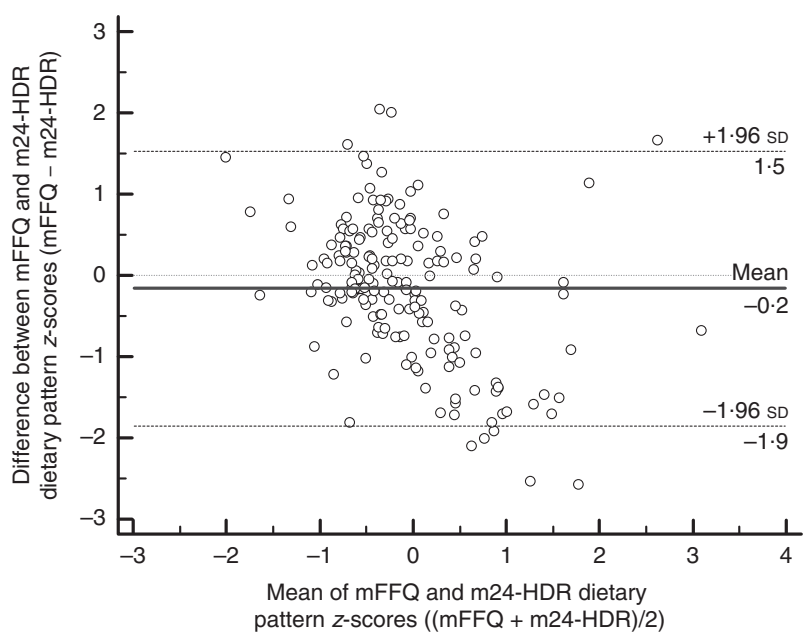

Fig. 5. Bland-Altman plots for 'beverage and alcohol' pattern $z$-scores derived from the mean of two FFQ (mFFQ) and mean of four 3 consecutive day 24-HDR (m24-HDR).

method was adopted as a reference method. For reducing the effect of difference in seasonal food availability and seasonal food preferences, twelve 24-HDR (one for 3-month intervals) were collected, which covered variability in food consumption during different seasons. Moreover, 3 consecutive day 24-HDR were administered for 2 weekdays and 1 weekend day in a usual week. Therefore, the influence of different diets between weekdays and weekends could be taken into consideration.

Although the methods of reproducibility and validity of dietary patterns were different, the obtained correlations in the present study were similar to those reported by other studies. In the first such study reported by Hu et al. ${ }^{(13)}$ in 1999, the corrected correlations between the two FFQ and two 1-week diet records (DR) ranged from 0.45 to 0.74 for the prudent and the Western patterns among 127 US males. The correlations for the factor scores between the two FFQ were 0.70 for the prudent pattern and 0.67 for the Western pattern. In 879 Danish men and 927 Danish women ${ }^{(14)}$, three (green, sweet and traditional) for men and two (green and sweet-traditional) patterns for women were identified in data from a FFQ and a 7-d DR, with corrected correlations ranging between 0.34 and 0.61 . Khani et $a l .{ }^{(15)}$ provided results with uncorrected correlations ranging between 0.41 and 0.73 for healthy, Western and drinker patterns identified using a FFQ and four 1-week DR in a random subgroup of 362 Swedish women. The coefficients of reproducibility were 0.63 (healthy pattern), 0.68 (Western pattern) and 0.73 (drinker pattern). Among 585 pregnant women in the $\mathrm{UK}^{(16)}$, the correlation coefficients ranged between 0.35 and 0.67 for the dietary patterns derived from a FFQ and a $4-\mathrm{d}$ food diary. In a subsample of 244 men and 254 women in Japan ${ }^{(11)}$, Pearson's correlation coefficients between the two FFQ ranged from 0.55 for the Western pattern in men and the prudent pattern in women to 0.77 for the traditional Japanese pattern in men. The corresponding values between 1-week DR and the FFQ ranged from 0.32 for the Western pattern in men to 0.63 for the traditional pattern in women. In 132 Iranian populations ${ }^{(17)}$, the ICC between factors scores of the two FFQ were 0.72 for the traditional and 0.80 for the Western pattern, and corrected correlations between FFQ2 and twelve 24-HDR were 0.48 for the traditional and 0.75 for the Western pattern. Loy \& Jan Mohamed ${ }^{(18)}$ found that Pearson's correlation coefficients between FFQ and three 24-HDR for healthy and less-healthy patterns were 0.59 and 0.63 , respectively, in 162 Malay pregnant women.

When the dietary pattern scores were classified into quartiles, a higher percentage of participants being classified into the same or adjacent quartile ( $>75 \%)$ and a low percentage into opposite quartile $(<5 \%)$ were shown in four dietary patterns in the present study, which demonstrated moderate agreement and lower misclassification between two FFQ and m24-HDR. The weighted $\kappa$ statistic, which overcame agreement by chance, depicted fair-to-good agreement for dietary patterns.

The Bland-Altman plot is a better method to illustrate the exact agreement between two different dietary assessment methods, which estimates the mean agreement and the $95 \%$ $\mathrm{LOA}^{(27)}$. A wide LOA indicates that the potential for large differences between methods and agreement is considered poor. The mean agreement was approximately equal to 0 for four patterns between FFQ and $\mathrm{m}-24 \mathrm{HR}$ in this study. The $95 \% \mathrm{LOA}$ for four dietary patterns were acceptable, in accordance with the results of previous studies ${ }^{(12,16-18)}$. Although the $95 \%$ LOA in the 'Chinese traditional' pattern was wider than those in other patterns, these differences were marginal.

The correlation coefficients, $\kappa$ statistics and percentage of agreement were higher and the $95 \%$ LOA were slight narrower for the 'animal and plant protein' pattern compared with the other three patterns; meanwhile, the percentage of misclassification was lower for the 'animal and plant protein' pattern than others. This may due to the fact that the 'animal and plant protein' pattern was rich in some usual food groups during 1 year (such as red meat, poultry meat, fish and shrimp, eggs, soya foods) and that the other three patterns included infrequent (nuts, sweets and desserts, and snacks in the 'nuts and sweets' pattern) or seasonal food groups (fresh fruits and vegetables in the 'Chinese traditional' pattern, and beer, wine and liquor in the 'beverage and alcohol' pattern).

Examining nutrient profiles is a useful way to compare dietary patterns from different dietary methods. Nutrient intakes are informative because they describe the product of a dietary pattern. As expected, correlations of our study were weaker between the FFQ and the m24-HDR; however, the directions of associations were consistent.

A major strength of the present study was the fact that there were no differences in baseline characteristics between the subsample in the present study and the entire population in the cross-sectional nutrition and health study; four similar dietary patterns were also identified in the overall sample. Therefore, as the subsample in the reproducibility and validity study were representative, the results can be generalised to the entire population. In addition, a high recruitment rate $(81.9 \%)$ and detailed data collected by trained interviewers were included.

There are several limitations to the present study. First, the sample size was relative small ( $n$ 203), which might have led to inadequate study power. However, some studies ${ }^{(18,33)}$ have 
Table 4. Pearson's correlation coefficients between dietary pattern scores and energy-adjusted nutrient intakes from the mean of four 3 consecutive day 24-HDR (m24-HDR) in the subsamples ( $n$ 203)

\begin{tabular}{|c|c|c|c|c|c|c|c|c|c|c|c|c|}
\hline \multirow[b]{2}{*}{ Nutrient intakes } & \multicolumn{3}{|c|}{ Animal and plant protein pattern } & \multicolumn{3}{|c|}{ Nuts and sweets pattern } & \multicolumn{3}{|c|}{ Chinese traditional pattern } & \multicolumn{3}{|c|}{ Beverage and alcohol pattern } \\
\hline & FFQ1 & FFQ2 & m24-HDR & FFQ1 & FFQ2 & m24-HDR & FFQ1 & FFQ2 & m24-HDR & FFQ1 & FFQ2 & m24-HDR \\
\hline Protein & $0.280^{*}$ & $0.359^{*}$ & $0.443^{*}$ & 0.004 & -0.004 & -0.040 & $-0.151^{\star}$ & -0.129 & 0.002 & 0.001 & -0.007 & 0.143 \\
\hline Total fat & $-0.605^{\star}$ & $-0.632^{\star}$ & $-0.736^{\star}$ & 0.103 & 0.043 & 0.040 & -0.018 & -0.016 & $0.154^{\star}$ & $0.184^{*}$ & $0 \cdot 166^{\star}$ & $0 \cdot 170^{*}$ \\
\hline Carbohydrates & $0.235^{\star}$ & $0.318^{\star}$ & $0.376^{\star}$ & 0.132 & 0.097 & 0.077 & -0.095 & 0.045 & 0.120 & 0.005 & -0.008 & 0.082 \\
\hline Fibre & 0.135 & $0.225^{*}$ & $0.292^{*}$ & -0.019 & -0.022 & -0.041 & $-0.146^{*}$ & -0.099 & -0.054 & -0.026 & -0.046 & 0.042 \\
\hline Cholesterol & $-0.694^{\star}$ & $-0.631^{*}$ & $-0.826^{\star}$ & 0.102 & 0.039 & 0.023 & -0.092 & -0.017 & 0.017 & $0 \cdot 140^{*}$ & $0.141^{*}$ & $0 \cdot 166^{*}$ \\
\hline Vitamin A & $0.140^{*}$ & $0.227^{\star}$ & $0.293^{\star}$ & -0.032 & -0.031 & -0.051 & $-0.159^{*}$ & $-0.201^{*}$ & -0.090 & -0.027 & -0.043 & 0.134 \\
\hline Carotene & 0.114 & $0.205^{\star}$ & $0.265^{\star}$ & -0.038 & -0.035 & -0.058 & $-0.160^{*}$ & $-0.207^{\star}$ & -0.094 & -0.034 & -0.054 & 0.125 \\
\hline Retinol & $0.655^{\star}$ & $0.579^{\star}$ & $0.773^{\star}$ & $0.139^{*}$ & 0.093 & $0 \cdot 156^{\star}$ & -0.002 & 0.022 & 0.024 & $-0.189^{\star}$ & $-0.253^{\star}$ & $0 \cdot 130$ \\
\hline Thiamine & $0.369^{\star}$ & $0.420^{*}$ & $0.515^{\star}$ & 0.045 & -0.112 & $-0.399^{\star}$ & -0.045 & 0.084 & $0.176^{\star}$ & $-0.290^{\star}$ & $-0.207^{\star}$ & $-0.220^{*}$ \\
\hline Riboflavin & $0.294^{\star}$ & $0.370^{\star}$ & $0.460^{*}$ & 0.002 & -0.012 & -0.027 & -0.121 & -0.114 & -0.023 & 0.067 & 0.068 & 0.133 \\
\hline Niacin & $0.225^{\star}$ & $0.319^{\star}$ & $0.391^{*}$ & -0.037 & -0.048 & -0.139 & $-0.197^{\star}$ & $-0.238^{\star}$ & -0.049 & 0.019 & -0.014 & $0.234^{*}$ \\
\hline Vitamin C & 0.087 & $0.171^{*}$ & $0.207^{\star}$ & -0.016 & 0.025 & -0.027 & $-0.305^{\star}$ & $-0.381^{*}$ & $-0.317^{\star}$ & 0.018 & 0.019 & $0.460^{*}$ \\
\hline Vitamin E & $0.210^{*}$ & $0.290^{*}$ & $0.373^{*}$ & 0.046 & 0.033 & 0.048 & -0.097 & -0.050 & -0.014 & 0.009 & -0.001 & 0.066 \\
\hline $\mathrm{Ca}$ & $0.188^{\star}$ & $0.273^{\star}$ & $0.334^{*}$ & -0.018 & 0.002 & -0.047 & $-0.156^{\star}$ & $-0.177^{\star}$ & -0.068 & -0.006 & 0.007 & 0.085 \\
\hline $\mathrm{P}$ & $0.404^{*}$ & $0.480^{\star}$ & $0.560^{\star}$ & 0.037 & 0.026 & -0.060 & $-0.156^{\star}$ & -0.053 & 0.068 & 0.038 & 0.035 & 0.115 \\
\hline $\mathrm{K}$ & $0.183^{\star}$ & $0.276^{*}$ & $0.340^{\star}$ & -0.026 & -0.030 & -0.064 & $-0.157^{\star}$ & $-0.142^{\star}$ & -0.044 & -0.018 & -0.039 & 0.099 \\
\hline $\mathrm{Na}$ & -0.059 & -0.040 & -0.037 & -0.134 & -0.083 & $-0.236^{*}$ & -0.118 & $-0.487^{\star}$ & $-0.514^{\star}$ & -0.095 & -0.055 & 0.077 \\
\hline $\mathrm{Mg}$ & $0 \cdot 176^{\star}$ & $0.261^{*}$ & $0.337^{\star}$ & -0.005 & -0.010 & -0.038 & $-0.139^{\star}$ & -0.126 & -0.036 & -0.009 & -0.023 & 0.086 \\
\hline $\mathrm{Fe}$ & $0.162^{*}$ & $0.252^{\star}$ & $0.321^{*}$ & -0.024 & -0.027 & -0.077 & $-0.164^{\star}$ & -0.004 & 0.003 & -0.021 & -0.040 & -0.064 \\
\hline $\mathrm{Zn}$ & $0.263^{\star}$ & $0.348^{\star}$ & $0.429^{\star}$ & -0.007 & -0.017 & -0.075 & $-0.159^{*}$ & $-0.146^{\star}$ & -0.010 & 0.017 & -0.001 & $0.151^{*}$ \\
\hline Se & $0.652^{\star}$ & $0.677^{\star}$ & $0.799^{\star}$ & $0 \cdot 146^{*}$ & 0.088 & 0.091 & -0.079 & -0.023 & 0.103 & $-0.137^{\star}$ & $-0.138^{\star}$ & $-0.187^{*}$ \\
\hline $\mathrm{Cu}$ & $0.156^{\star}$ & $0.241^{*}$ & $0.311^{*}$ & 0.002 & -0.002 & -0.026 & -0.132 & -0.131 & -0.033 & -0.018 & -0.023 & 0.086 \\
\hline $\mathrm{Mn}$ & 0.115 & $0.206^{*}$ & $0.267^{*}$ & -0.037 & -0.036 & -0.060 & $-0.157^{\star}$ & $-0.250^{*}$ & $-0.173^{*}$ & -0.035 & -0.055 & 0.099 \\
\hline
\end{tabular}


suggested that the generally accepted sample size is seven participants per food group for FA. Second, in the absence of an absolute gold standard for dietary assessment, we chose dietary recalls as a reference method. This method was advantageous in its ability to collect actual intake on specific days. However, dietary recalls might also be subject to recall bias, erroneous recording and potential changes in eating behaviour, leading to over-estimating or under-estimating food intake. Therefore, we attempted to minimise weakness by checking dietary recalls by following-up incomplete or ambiguous information directly with respondents. Moreover, four 3 consecutive day 24-HDR were shown to be sufficient to capture seasonal variations in food intake. Third, the analysis of reproducibility and validity was confined to adults aged 31-80 years. It is unclear whether our findings can be applied to children, adolescents and younger adults. Finally, the total variance explained by the four dietary patterns derived from FFQ1, FFQ2 and m24-HDR was 40.0, 44.9, 32.4\%, respectively, suggesting the existence of minor dietary patterns, which were less interpretable and highly variable; therefore, they were not presented in this study.

In conclusion, our study indicated a good reproducibility and a reasonable validity of the major dietary patterns identified by FA using data from a FFQ and dietary recalls among Chinese populations, suggesting that FFQ data provided useful information on dietary patterns. Dietary pattern might be used in nutrition epidemiology as a complementary approach to traditional analysis and is appropriate to examine the diet-disease association.

\section{Acknowledgements}

The authors are grateful to all the dedicated fieldworkers who took part in the survey and all participants who facilitated the survey implementation at each community.

The present study was supported by Nanjing Municipal Medical Science and Technique Development Foundation, China (grant no. 2012-YKK12166).

X. H., Q. Y. and F. X. contributed to the study design and data analysis; X. H., Q. Y., F. X., Z. W., H. Y., X. C., H. Z., C. W., W. C., Y. L. and L. S. contributed to data collection; Z. W., H. Y., X. C., H. Z., C. W., W. C., Y. L. and L. S. were responsible for manuscript revision; Y. W. was responsible for power calculation and language editing; and X. H., Q. Y., F. X. and Y. W. contributed to manuscript writing.

The authors declare that there are no conflicts of interest.

\section{References}

1. Hu FB (2002) Dietary pattern analysis: a new direction in nutritional epidemiology. Curr Opin Lipidol 13, 3-9.

2. Newby PK \& Tucker KL (2004) Empirically derived eating patterns using factor or cluster analysis: a review. Nutr Rev $\mathbf{6 2}$, 177-203.

3. DiBello JR, Kraft P, McGarvey ST, et al. (2008) Comparison of 3 methods for identifying dietary patterns associated with risk of disease. Am J Epidemiol 168, 1433-1443.

4. Reedy J, Wirfält E, Flood A, et al. (2010) Comparing 3 dietary pattern methods - cluster analysis, factor analysis, and index analysis - with colorectal cancer risk: the NIH-AARP diet and health study. Am J Epidemiol 171, 479-487.

5. Martinez ME, Marshall JR \& Sechrest L (1998) Invited commentary: factor analysis and the search for objectivity. Am J Epidemiol 148, 17-19.

6. Villegas R, Yang G, Gao YT, et al. (2010) Dietary patterns are associated with lower incidence of type 2 diabetes in middleaged women: the Shanghai women's health study. Int $J$ Epidemiol 39, 889-899.

7. Shim JS, Oh K \& Kim HC (2014) Dietary assessment methods in epidemiologic studies. Epidemiol Health 36, e2014009 (Review).

8. Villegas R, Yang G, Liu D, et al. (2007) Validity and reproducibility of the food-frequency questionnaire used in the Shanghai men's health study. Br J Nutr 97, 993-1000.

9. Xia W, Sun C, Zhang L, et al. (2011) Reproducibility and relative validity of a food frequency questionnaire developed for female adolescents in Suihua, North China. PLOS ONE 6, e19656.

10. Zhuang M, Yuan Z, Lin L, et al. (2012) Reproducibility and relative validity of a food frequency questionnaire developed for adults in Taizhou, China. PLOS ONE 7, e48341.

11. Nanri A, Shimazu T, Ishihara J, et al. (2012) Reproducibility and validity of dietary patterns assessed by a food frequency questionnaire used in the 5-year follow-up survey of the Japan. Public health center-based prospective study. J Epidemiol 22, 205-215.

12. Okubo H, Murakami K, Sasaki S, et al. (2010) Relative validity of dietary patterns derived from a self-administered diet history questionnaire using factor analysis among Japanese adults. Public Health Nutr 13, 1080-1089.

13. Hu FB, Rimm E, Smith-Warner SA, et al. (1999) Reproducibility and validity of dietary patterns assessed with a food-frequency questionnaire. Am J Clin Nutr 69, 243-249.

14. Togo P, Heitmann BL, Sorensen TI, et al. (2003) Consistency of food intake factors by different dietary assessment methods and population groups. Br J Nutr 90, 667-678.

15. Khani BR, Ye W, Terry P, et al. (2004) Reproducibility and validity of major dietary patterns among Swedish women assessed with a food-frequency questionnaire. J Nutr 134, 1541-1545.

16. Crozier SR, Inskip HM, Godfrey KM, et al. (2008) Dietary patterns in pregnant women: a comparison of food frequency questionnaires and $4 \mathrm{~d}$ prospective diaries. Br J Nutr 99, 869-875.

17. Asghari G, Rezazadeh A, Hosseini-Esfahani F, et al. (2012) Reliability, comparative validity and stability of dietary patterns derived from an FFQ in the Tehran lipid and glucose study. Br J Nutr 108, 1109-1117.

18. Loy SL \& Jan Mohamed HJ (2013) Relative validity of dietary patterns during pregnancy assessed with a food frequency questionnaire. Int J Food Sci Nutr 64, 668-673.

19. Ye Q, Hong X, Wang Z, et al. (2016) Reproducibility and validity of an FFQ developed for adults in Nanjing, China. BrJ Nutr 115, 887-894.

20. Qin Y, Melse-Boonstra A, Yuan B, et al. (2012) Zinc biofortification of rice in China: a simulation of zinc intake with different dietary patterns. Nutrients $\mathbf{4}, 517-528$.

21. Yang Y (2005) Chinese Food Composition Table 2004. Beijing: Peking University Medical Press.

22. Willett W \& Stampfer MJ (1986) Total energy intake: implications for epidemiologic analyses. Am J Epidemiol 124, 17-27.

23. Shi Z, Hu X, Yuan B, et al. (2008) Vegetable-rich food pattern is related to obesity in China. Int J Obes (Lond) 32, 975-984.

24. Hatcher LA (1994) Step-By-Step Approach to Using SAS for Factor Analysis and Structural Equation Modeling. Cary, NC: SAS Institute. 
25. Gibson RS (2005) Principles of Nutritional Assessment, 2nd ed. New York, NY: Oxford University Press.

26. Willett W \& Lenart E (1998) Reproducibility and validity of food-frequency questionnaires. In Nutritional Epidemiology, 2nd ed. pp 101-147 [W Willett, editor]. Oxford: Oxford University Press.

27. Bland JM \& Altman DG (1986) Statistical methods for assessing agreement between two methods of clinical measurement. Lancet 1, 307-310.

28. Willett WC (1998) Nutritional Epidemiology, 2nd ed. New York: Oxford University Press.

29. Livingstone MBE \& Black AE (2003) Markers of the validity of reported energy intake. J Nutr 133, 895S-920S.
30. Luo YZ, Chen XW, Miu GZ, et al. (2009) Association between hypertension and dietary patterns in residents of Jiangyin city. Chin J Public Health 25, 314-316 (In Chinese).

31. Dai X, He P, Zhang YF, et al. (2010) Dietary pattern of Shanghai community-based middle and aged women. $J$ Hyg Res 39, 472-477 (In Chinese).

32. Odegaard AO, Koh WP, Butler LM, et al. (2011) Dietary patterns and incident type 2 diabetes in Chinese men and women: the Singapore Chinese health study. Diabetes Care 34, 880-885.

33. Floyd F \& Widaman K (1995) Factor analysis in the development and refinement of clinical assessment instruments. Psychol Assess 7, 286-299.

\section{Appendix 1}

The twenty-eight food groups used in the dietary pattern analysis

\begin{tabular}{|c|c|}
\hline Food groups & Food items \\
\hline 1. Rice and products & White rice, millet, porridge, rice flour \\
\hline 2. Wheat and products & Noodle, dumpling, bread, steamed bread, wonton, twist bread \\
\hline 3. Potatoes & Potatoes, Chinese yam, sweet potato, taro \\
\hline 4. Other grains and products & Sorghum, maize \\
\hline 5. Fried food & Deep-fried dough sticks, deep-fried dough cake, other fried food \\
\hline 6. Livestock meats & Beef, steak, lamb, pork, meatballs, meatloaf, ham \\
\hline 7. Poultry meats & Chicken, duck, goose \\
\hline 8. Processed meats & Sausage, bacon, hot dogs \\
\hline 9. Fish and shrimp & Carp, grass carp, silver carp, herring, shrimp \\
\hline 10. Eggs & Eggs \\
\hline 11. High-fat dairy products & Butter, cheese, whole milk, whole yogurt, ice cream \\
\hline 12. Low-fat dairy products & Low-fat milk, reduced-fat (medium) milk, low-fat yogurt fat yogurt \\
\hline 13. Bean curd & Bean curd \\
\hline 14. Dry beans & Red bean, green beans, black beans, soyabean, bean curd \\
\hline 15. Other soyabean products & Bean sprouts, soya chicken, bean curd sheet, soyabean milk \\
\hline 16. Fresh vegetables & $\begin{array}{l}\text { Spinach, cucumber, Chinese cabbage, lettuce, broccoli, greens, celery, water spinach, shepherd purse, carrots, } \\
\text { tomatoes, onion, eggplant, yellow squash, mushrooms, green pepper }\end{array}$ \\
\hline 17. Pickled vegetables & Pickled vegetables \\
\hline 18. Dry vegetables & Dried mushroom, dried black fungus, kelp, laver \\
\hline 19. Fresh fruits & Oranges, bananas, apples, strawberries, grapes, peaches, pears, kiwifruit, melons, watermelon \\
\hline 20. Juice & Fruit or vegetable juice \\
\hline 21. Sodas & Cola, other carbonated beverage \\
\hline 22. Tea or coffee & Red or green tea, coffee \\
\hline 23. Beer & Beer, regular or light \\
\hline 24. Liquor & Liquor \\
\hline 25. Wine & Red or white wine \\
\hline 26. Sweets and desserts & Candy, cookie, chocolate, brownies, cake, pie, pastry \\
\hline 27. Nuts & Peanut, walnut, melon seed, cashew, other nuts \\
\hline 28. Other snacks & Potato chips or maize chips, crackers, popcorn \\
\hline
\end{tabular}




\section{Appendix 2}

Comparison of participants in the reliability and validity study with those in the cross-sectional survey (Mean values and standard deviations)

\begin{tabular}{|c|c|c|c|c|c|}
\hline \multirow[b]{2}{*}{ Variables } & \multicolumn{2}{|c|}{$\begin{array}{l}\text { Subsample } \\
(n \text { 203) }\end{array}$} & \multicolumn{2}{|c|}{$\begin{array}{c}\text { Total sample } \\
(n \text { 2030) }\end{array}$} & \multirow[b]{2}{*}{$P$} \\
\hline & Mean & SD & Mean & SD & \\
\hline Age at recruitment (years) & $50 \cdot 4$ & $12 \cdot 2$ & 51.5 & $12 \cdot 3$ & 0.224 \\
\hline $\operatorname{BMI}\left(\mathrm{kg} / \mathrm{m}^{2}\right)$ & 23.1 & $2 \cdot 8$ & 23.5 & 3.1 & 0.077 \\
\hline $\operatorname{Sex}(\%)$ & & & & & 0.639 \\
\hline Male & \multicolumn{2}{|c|}{$48 \cdot 8$} & \multicolumn{2}{|c|}{50.5} & \\
\hline Female & \multicolumn{2}{|c|}{$51 \cdot 2$} & \multicolumn{2}{|c|}{49.5} & \\
\hline Marriage (\%) & & & & & 0.259 \\
\hline Married & \multicolumn{2}{|c|}{$92 \cdot 2$} & \multicolumn{2}{|c|}{$89 \cdot 7$} & \\
\hline Unmarried/divorced/widowed & \multicolumn{2}{|c|}{7.8} & \multicolumn{2}{|c|}{$10 \cdot 3$} & \\
\hline Education (\%) & & & & & 0.080 \\
\hline Primary school and lower & \multicolumn{2}{|c|}{20.5} & \multicolumn{2}{|c|}{$28 \cdot 2$} & \\
\hline Junior high school & \multirow{2}{*}{\multicolumn{2}{|c|}{$32 \cdot 2$}} & \multicolumn{2}{|c|}{$32 \cdot 2$} & \\
\hline Senior high school & \multirow{2}{*}{\multicolumn{2}{|c|}{$\begin{array}{l}24.4 \\
22.9\end{array}$}} & \multirow{2}{*}{\multicolumn{2}{|c|}{$20 \cdot 6$}} & \\
\hline College and higher & & & $19 \cdot 0$ & & \\
\hline Occupation (\%) & \multicolumn{2}{|c|}{$22 \cdot 9$} & & & 0.157 \\
\hline Manual labourers & \multicolumn{2}{|c|}{$42 \cdot 4$} & \multicolumn{2}{|c|}{$46 \cdot 2$} & \\
\hline Service staff & \multicolumn{2}{|c|}{$6 \cdot 8$} & \multicolumn{2}{|c|}{6.5} & \\
\hline Mental labourers & \multirow{2}{*}{\multicolumn{2}{|c|}{$\begin{array}{r}25.9 \\
24.9\end{array}$}} & \multicolumn{2}{|c|}{$19 \cdot 4$} & \\
\hline Others & & & \multicolumn{2}{|c|}{28.0} & \\
\hline Current smokers (\%) & \multicolumn{2}{|c|}{$24 \cdot 9$} & & & 0.918 \\
\hline Yes & \multicolumn{2}{|c|}{$22 \cdot 0$} & \multicolumn{2}{|c|}{$22 \cdot 3$} & \\
\hline No & \multicolumn{2}{|c|}{78.0} & \multicolumn{2}{|c|}{$77 \cdot 7$} & \\
\hline Current drinkers (\%) & & & & & 0.202 \\
\hline Yes & \multicolumn{2}{|c|}{28.8} & & & \\
\hline No & & & & & \\
\hline
\end{tabular}

\section{Appendix 3}

Factor-loading matrix for the four major dietary patterns* identified using factor analysis in the overall samples $(n$ 2030)

\begin{tabular}{|c|c|c|c|c|}
\hline Food groups & Animal and plant protein & Nuts and sweets & Chinese traditional & Beverage and alcohol \\
\hline Poultry meats & $0.739 \dagger$ & 0.072 & 0.015 & 0.286 \\
\hline Fish and shrimp & $0.656 \dagger$ & 0.038 & 0.124 & 0.198 \\
\hline Bean curd & $0.365 \dagger$ & -0.016 & 0.232 & $0.363 \dagger$ \\
\hline Dry vegetables & $0.300 \dagger$ & 0.214 & 0.262 & $-0 \cdot 164$ \\
\hline Livestock meats & $0.724 \dagger$ & 0.098 & 0.010 & 0.246 \\
\hline Dry bean & $0.576 \dagger$ & 0.196 & 0.016 & 0.084 \\
\hline Other soyabean products & $0.460 \dagger$ & 0.231 & -0.031 & 0.141 \\
\hline Eggs & $0.474 \dagger$ & 0.022 & 0.103 & $-0 \cdot 115$ \\
\hline Fresh fruits & $0.503 \dagger$ & 0.231 & 0.020 & -0.216 \\
\hline Low-fat dairy products & $0.359 \dagger$ & 0.267 & 0.145 & -0.277 \\
\hline Tea or coffee & 0.228 & 0.092 & 0.001 & 0.067 \\
\hline Nuts & 0.121 & $0.571 \dagger$ & 0.113 & $-0 \cdot 166$ \\
\hline Sweets and desserts & 0.054 & $0.458 \dagger$ & $-0 \cdot 106$ & 0.014 \\
\hline Snacks & 0.042 & $0.613 \dagger$ & 0.019 & -0.072 \\
\hline Other grains and products & 0.142 & -0.067 & $0.609 \dagger$ & -0.214 \\
\hline Potatoes & $0.338 \dagger$ & -0.046 & $0.605 t$ & 0.020 \\
\hline Fresh vegetables & -0.254 & -0.091 & $0.419 \dagger$ & 0.225 \\
\hline Fried food & -0.104 & -.006 & $0.466 \dagger$ & 0.232 \\
\hline High-fat dairy products & 0.188 & 0.116 & $0.399 \dagger$ & -0.299 \\
\hline Wheat and products & 0.210 & -0.053 & $0.427 \dagger$ & -0.036 \\
\hline Rice and products & 0.130 & $-0 \cdot 101$ & $0.563 \dagger$ & -0.096 \\
\hline Pickled vegetables & -0.141 & 0.057 & $0.421 \dagger$ & 0.031 \\
\hline Sodas & -0.028 & 0.196 & 0.033 & $0.562 \dagger$ \\
\hline Juice & 0.085 & 0.015 & 0.010 & $0.454 \dagger$ \\
\hline Beer & 0.007 & 0.044 & -0.010 & $0.397 \dagger$ \\
\hline Wine & 0.159 & 0.099 & 0.038 & $0.380 \dagger$ \\
\hline Processed meats & 0.147 & 0.080 & 0.147 & $0.373 \dagger$ \\
\hline Liquor & - & 0.022 & 0.028 & $0.426 \dagger$ \\
\hline Eigenvalue & 3.378 & 1.864 & 2.493 & 1.820 \\
\hline Variance explained (\%) & 11.3 & $6 \cdot 2$ & 8.3 & 6.1 \\
\hline Total variance $(\%)$ & \multicolumn{4}{|c|}{31.9} \\
\hline
\end{tabular}

* The test for suitability of factor analysis: the Kaiser-Meyer-Olkin measure of sampling adequacy was 0.702 for FFQ1 and $P$ values for Bartlett's test of sphericity was $<0.001$.

$\dagger$ The factor loadings were $>0 \cdot 30$. 